\title{
Is finger-counting necessary for the development of arithmetic abilities?
}

\section{Virginie Crollen*, Xavier Seron and Marie-Pascale Noël}

Centre de Neuroscience Système et Cognition, Institut de Recherche en Sciences Psychologiques, Université Catholique de Louvain, Louvain-la-Neuve, Belgium *Correspondence: virginie.crollen@uclouvain.be

In the literature on numerical cognition, it is generally assumed that fingers play a functional role in the development of a mature counting system (Gelman and Gallistel, 1978; Fuson et al., 1982; Fuson, 1988; Butterworth, 1999a,b, 2005). Indeed, fingers have been assumed to contribute to: (1) giving an iconic representation of numbers (Fayol and Seron, 2005); (2) keeping track of the number words uttered while reciting the counting sequence (Fuson et al., 1982); (3) sustaining the induction of the one-toone correspondence principle (Alibali and DiRusso, 1999) by helping children to coordinate the processes of tagging (i.e., attribution of a counting word to each item) and partitioning (i.e., isolating the items already counted from those which remained to be counted; Gelman and Gallistel, 1978); (4) sustaining the assimilation of the stableorder principle (i.e., numerical labels have to be enumerated in the same order across counting sequences) by supporting the emergence of a routine to link fingers to objects in a sequential, culture-specific stable order (Wiese, 2003a,b); (5) sustaining the comprehension of the cardinality principle (i.e., the last number word uttered while counting determines the total number of objects in a set) by leading children to always reach the same finger when counting to a specific number (Fayol and Seron, 2005); (6) prompting the understanding of the 10-base numerical system (as on our hands we represent numbers as a sum and/ or a multiple of 10); and (7) sustaining the realization of basic arithmetic operations (Baroody, 1987; Fuson and Kwon, 1992; Geary, 1994; Ifrah, 2000).

In line with these assumptions, several studies have reported the existence of a close connection between finger representation and number processing (for a review, see Moeller et al., submitted). At a developmental level, for example, performance on finger discrimination tasks was shown to be a good predictor of arithmetic abilities (Fayol et al., 1998; Noël, 2005).
Moreover, the specific sub-base-five structure of the finger-counting system (i.e., the representation of numbers larger than 5 always includes a full hand pattern) was shown to influence numerical processing in infants, hearing adults, and deaf signers (Iversen et al., 2006; Domahs et al., 2008, 2010). Finally, brain imaging studies suggested that the finger schema could rely on the same neuroanatomical substrate (i.e., parietal network) as the processing of numbers (Pesenti et al., 2000; Piazza et al., 2002; Pinel et al., 2004). Accordingly, some authors have suggested that fingers may be the "missing tool" (Andres et al., 2008) that sustains the assimilation of basic numerical abilities or the "missing link" (Fayol and Seron, 2005) that permits the connection between non-symbolic numerosities and symbolic arithmetic.

In this paper, we will not contest the empirical evidence showing that the use of fingers to represent numbers has a very important impact on numerical cognition. Rather, we will address the question of whether finger-counting is part of a necessary stage for the development of numerical cognition and whether its use is spontaneous in every human child.

If fingers constitute a first and obligatory step in numerical development, then one might expect that children first represent quantities with their fingers before being able to represent them with number words. Similarly, we might expect that during the first developmental stages, children would be more accurate to represent numerosities with their fingers than with number words. To our knowledge, no systematic longitudinal data have been reported on the developmental chronology of fingers use versus number word use. However, interesting transversal observations have been recently published by Nicoladis et al. (2010) who presented either hand shape or number words (from 1 to 10 ) to 2 - to 5 -year old children and asked them to put that number of toys in a box. No difference was seen between these two presentation modalities for 2- and 3-year olds who performed equally badly in the two conditions. Yet, for 4- and 5-year olds, performance was actually better with number words than with hand shapes. The authors also presented collections of toys to the children who were asked to say the corresponding number word or to show the correct number of fingers. Again, performance was better with words than with hand shapes. This result does therefore not support the idea that the symbolic numerical system is rooted in our bodily experience. However, it is possible that praxic difficulties contribute to explaining the poor performance obtained by the children in the second task, when required to show a finger configuration corresponding to the number of toys. However, this does not explain the weaker performance in the first task and it also indicates that the use of fingers does not precede the use of language.

Another interesting opportunity to examine whether the finger code facilitates the development of the concept of exact number is the study of homesigners (i.e., deaf children who do not have access to a model for signed language but who nevertheless develop their own gestures to communicate). In their study, Spaepen et al. (2011) examined the numerical abilities of adult deaf homesigners who use their fingers to communicate about numbers. For sets containing more than three items, the number of fingers used to indicate the number of objects in a set was close but most of the time not equal to the number of items to represent. Similarly, homesigners were not able to produce a set of the same number as another one. In some situations, they used their fingers to establish one-to-one correspondence and thus achieved greater accuracy than with pure approximation but they did not use this strategy in all conditions. For instance, they did not use it when asked to produce a set of the same number as a series of sequential events. Importantly, in all these tasks, homesigners performed 
more poorly than deaf individuals who had learned a sign language. So, despite the fact that homesigners used their fingers to communicate about numbers, they did not consistently and accurately represent the cardinality of sets containing more than three items. So, when fingers configurations used to represent numbers are not embedded in a counting routine, their iconic structure seems insufficient to allow the development of an exact representation of large numbers.

The second question we will address in this paper is whether finger use is spontaneous or whether it requires some modeling. In several contexts, including the resolution of basic additions and subtractions (Geary, 1994), we use fingers to keep track of our counting. We might thus wonder whether this is a spontaneous practice or whether we develop this strategy because we have seen others doing it. Recently, Crollen et al. (2011) compared the spontaneous use of fingers to count and to represent numerical quantities in blind and sighted children. Although these two groups of participants did not differ in terms of basic finger discrimination abilities, blind children used finger-counting strategies significantly less frequently than their sighted peers. Moreover, despite this difference in finger use, blind and sighted children achieved quite similar level of performance in several enumeration tasks. In fact, blind children had weaker performance than sighted controls only when the tasks were very heavy in terms of verbal working memory resources (counting two series in parallel or counting with concomitant articulatory suppression). These data therefore suggest that finger-counting is a useful tool to alleviate the working memory load but not a necessary tool for the emergence of good counting skills. Furthermore, when explicitly asked to count and show quantity with their fingers, the majority of blind children showed unconventional and unstable (changing from trial to trial) configurations of fingers, suggesting that they had used their fingers for the first time. Yet, in a simple addition task, both groups performed equally well. These data suggest that, without the opportunity to watch others using their fingers, many blind children did not spontaneously use their fingers to count or to show numbers.
Yet, they were able to keep track of their counting in another manner and to learn basic arithmetic equally as well as sighted controls. These results thus indicate that finger use is not universal or spontaneous but requires some modeling.

In summary, fingers have been assumed to play a crucial role in the development of a mature counting system. However, in this paper, we have presented empirical evidence that constrains this hypothesis. First, in typically developing children, the use of fingers does not precede the use of language. Second, for hearing children, the iconicity of hand shape does not seem to be an advantage for representing numbers. Third, if not embedded in a counting routine, use of finger configuration to represent numbers is not sufficient to allow the development of an exact representation of large numbers. Fourth, models of fingercounting are culturally determined (see Bender and Beller, submitted) and use of fingers is rare when these models are not available (as in blind children). Finally, children who do not use their fingers to count and represent numbers do not show atypical or delayed numerical development. Accordingly, we argue that finger-counting is not a necessary step for numerical development. However, it is undoubtedly a very useful tool (Beller and Bender, 2011; Di Luca and Pesenti, submitted) that allows, among other things, the working memory load to be alleviated and thus perform better in complex numerical tasks. Thus, explicit teaching of this useful tool might be considered in kindergarten, especially in populations where natural access to the social transmission of this system is problematic (e.g., in blind populations).

\section{REFERENCES}

Alibali, M. W., and DiRusso, A. A. (1999). The function of gesture in learning to count: more than keeping track. Cogn. Dev. 14, 37-56.

Andres, M., Di Luca, S., and Pesenti, M. (2008). Fingercounting: the missing tool? Behav. Brain Sci. 31, 642-643.

Baroody, A. J. (1987). Children's Mathematical Thinking: A Developmental Framework for Preschool, Primary, and Special Education Teachers. New York: Teacher's College.

Beller, S., and Bender, A. (2011). Explicating numerical information: When and how fingers support (or hinder) number comprehension and handling. Front. Psychology. doi: 10.3389/fpsyg.2011.00214

Butterworth, B. (1999a). The Mathematical Brain. London: Macmillan.
Butterworth, B. (1999b). A head for figures. Science 284, 928-929.

Butterworth, B. (2005). The development of arithmetical abilities. J. Child Psychol. Psychiatry 46, 3-18.

Crollen, V., Mahe, R., Collignon, O., and Seron, X. (2011). The role of vision in the development of fingernumber interactions: finger-counting and fingermontring in blind children. J. Exp. Child. Psychol. 109, 525-539.

Domahs, F., Krinzinger, H., and Willmes, K. (2008). Mind the gap between both hands: evidence for internal finger-based number representations in children's mental calculation. Cortex 44, 359-367.

Domahs, F., Moeller, K., Huber, S., Willmes, K., and Nuerk, H. C. (2010). Embodied numerosity: implicit handbased representation influence symbolic number processing across cultures. Cognition 116, 251-266.

Fayol, M., Barrouillet, P., and Marinthe, C. (1998). Predicting mathematical achievement from neuropsychological performance: a longitudinal study. Cognition 68, 63-70.

Fayol, M., and Seron, X. (2005). “About numerical representations: insights from neuropsychological, experimental and developmental studies," in Handbook of Mathematical Cognition, ed. J. I. D. Campbell (New York: Psychology Press), 3-22.

Fuson, K. C. (1988). Children's Counting and Concepts of Number. New York: Springer-Verlag.

Fuson, K. C., and Kwon, Y. (1992). "Learning addition and subtraction: effects of number word and other cultural tools," in Pathways to Number, eds J. Bideau, C. Meljac, and J. P. Fisher (Hillsdale, NJ: Erlbaum), 351-374.

Fuson, K. C., Richards, J., and Briars, D. J. (1982). “The acquisition and elaboration of the number word sequence," in Children's Logical and Mathematical Cognition, ed. C. J. Brainerd (New York: SpringerVerlag), 33-92.

Geary, D. C. (1994). "Developing arithmetical skills," in Children's mathematical development: Research and Practical Applications, ed. D. C. Geary (Washington, DC: American Psychological Association), 37-93.

Gelman, R., and Gallistel, C. R. (1978). The Child's Understanding of Number. Cambridge, MA: Harvard University Press.

Ifrah, G. (2000). The Universal History of Numbers. London: Harvill.

Iversen, W., Nuerk, H. C., Jager, L., and Willmes, K. (2006). The influence of an external symbol system on number parity representation, or what's odd about 6? Psychon. Bull. Rev. 13, 730-736.

Nicoladis, E., Pika, S., and Marentette, P. (2010). Are number gestures easier than number words for preschoolers? Cogn. Dev. 25, 247-261.

Noël, M. P. (2005). Finger gnosia: a predictor of numerical abilities in children? Child Neuropsychol. 11, 413-430.

Pesenti, M., Thioux, M., Seron, X., and De Volder, A. (2000). Neuroanatomical substrates of Arabic number processing, numerical comparison, and simple addition: a PET study. J. Cogn. Neurosci. 12, 461-479.

Piazza, M., Mechelli, A., Butterworth, B., and Price, C. J. (2002). Are subitizing and counting implemented as separate or functionally overlapping processes? Neuroimage 15, 435-446.

Pinel, P., Piazza, M., Le Bihan, D., and Dehaene, S. (2004). Distributed and overlapping cerebral 
representations of number, size, and luminance during comparative judgments. Neuron 41, 983-993.

Spaepen, E., Coppola, M., Spelke, E. S., Carey, S. E., and Goldin-Meadow, S. (2011). Number without a language model. PNAS 108, 3163-3168.

Wiese, H. (2003a). Iconic and non-iconic stages in number development: the role of language. Trends Cogn. Sci. (Regul. Ed.) 7, 385-390.
Wiese, H. (2003b). Numbers, Language, and the Human Mind. Cambridge, MA: Cambridge University Press.

Received: 21 June 2011; accepted: 04 September 2011; published online: 26 September 2011.

Citation: Crollen V, Seron X and Noël M-P (2011)

Is finger-counting necessary for the development of arithmetic abilities? Front. Psychology 2:242. doi: 10.3389/ fpsyg.2011.00242
This article was submitted to Frontiers in Cognition, a specialty of Frontiers in Psychology.

Copyright (c) 2011 Crollen, Seron and Noël. This is an open-access article subject to a non-exclusive license between the authors and Frontiers Media SA, which permits use, distribution and reproduction in other forums, provided the original authors and source are credited and other Frontiers conditions are complied with. 